\title{
Clinical dyslipidaemia is associated with changes in the lipid composition and inflammatory properties of apolipoprotein-B-containing lipoproteins from women with type 2 diabetes
}

\author{
M. Ståhlman • H. T. Pham • M. Adiels • T. W. Mitchell • \\ S. J. Blanksby • B. Fagerberg • K. Ekroos • J. Borén
}

Received: 11 October 2011 / Accepted: 12 December 2011 / Published online: 18 January 2012

(C) Springer-Verlag 2012

\begin{abstract}
Aims/hypothesis The aim of this study was to use lipidomics to determine if the lipid composition of apolipoprotein-Bcontaining lipoproteins is modified by dyslipidaemia in type 2 diabetes and if any of the identified changes potentially have biological relevance in the pathophysiology of type 2 diabetes. Methods VLDL and LDL from normolipidaemic and dyslipidaemic type 2 diabetic women and controls were isolated and quantified with HPLC and mass spectrometry. A detailed molecular characterisation of VLDL triacylglycerols (TAG) was also performed using the novel ozone-induced dissociation method, which allowed us to distinguish vaccenic acid (C18:1 $n$-7) from oleic acid (C18:1 $n$-9) in specific TAG species. Results Lipid class composition was very similar in VLDL and LDL from normolipidaemic type 2 diabetic and control
\end{abstract}

Electronic supplementary material The online version of this article (doi:10.1007/s00125-011-2444-6) contains peer-reviewed but unedited supplementary material, which is available to authorised users.

M. Ståhlman · M. Adiels · B. Fagerberg · J. Borén $(\bowtie)$

Sahlgrenska Centre for Cardiovascular and Metabolic Research/

Wallenberg Laboratory and Department of Molecular and Clinical

Medicine, Sahlgrenska University Hospital,

S-413 45 Gothenburg, Sweden

e-mail: Jan.Boren@wlab.gu.se

H. T. Pham • S. J. Blanksby

School of Chemistry, University of Wollongong,

Wollongong, NSW, Australia

T. W. Mitchell

School of Health Sciences, University of Wollongong,

Wollongong, NSW, Australia

K. Ekroos

Zora Biosciences Oy,

Espoo, Finland participants. By contrast, dyslipidaemia was associated with significant changes in both lipid classes (e.g. increased diacylglycerols) and lipid species (e.g. increased C16:1 and $\mathrm{C} 20: 3$ in phosphatidylcholine and cholesteryl ester and increased C16:0 [palmitic acid] and vaccenic acid in TAG). Levels of palmitic acid in VLDL and LDL TAG correlated with insulin resistance, and VLDL TAG enriched in palmitic acid promoted increased secretion of proinflammatory mediators from human smooth muscle cells.

Conclusions We showed that dyslipidaemia is associated with major changes in both lipid class and lipid species composition in VLDL and LDL from women with type 2 diabetes. In addition, we identified specific molecular lipid species that both correlate with clinical variables and are proinflammatory. Our study thus shows the potential of advanced lipidomic methods to further understand the pathophysiology of type 2 diabetes.

Keywords Apolipoprotein B · LDL · Lipidomics · Lipids . Mass spectrometry VLDL

$\begin{array}{ll}\text { Abbreviations } \\ \text { Apo } & \text { Apolipoprotein } \\ \text { CE } & \text { Cholesteryl ester } \\ \text { CER } & \text { Ceramide } \\ \text { CID } & \text { Collision-induced dissociation } \\ \text { DAG } & \text { Diacylglycerol } \\ \text { FAME } & \text { Fatty acid methyl esters } \\ \text { FC } & \text { Free (non-esterified) cholesterol } \\ \text { FID } & \text { Flame ionisation detector } \\ \text { IDL } & \text { Intermediate density lipoprotein } \\ \text { LPC } & \text { Lysophosphatidylcholine } \\ \text { OzID } & \text { Ozone-induced dissociation } \\ \text { PC } & \text { Phosphatidylcholine }\end{array}$




$\begin{array}{ll}\text { SM } & \text { Sphingomyelin } \\ \text { SMase } & \text { Sphingomyelinase } \\ \text { TAG } & \text { Triacylglycerol } \\ \text { UKPDS } & \text { UK Prospective Diabetes Study }\end{array}$

\section{Introduction}

Individuals with type 2 diabetes have an increased risk of cardiovascular events [1]. Although the mechanisms behind this increased risk are still not fully understood, dyslipidaemia is common in patients with type 2 diabetes and is an established risk factor for cardiovascular disease [2]. Fatty liver has evolved as a key player in the pathogenesis of dyslipidaemia and we have shown a strong relationship between increased liver fat, insulin resistance and the overproduction of large (triacylglycerol [TAG]-rich) VLDL particles [3, 4]. The increased secretion of large VLDL particles initiates a sequence of lipoprotein changes, resulting in additional abnormalities observed in dyslipidaemia such as low levels of HDL and the appearance of small dense LDL [5]. In addition, hepatic uptake of VLDL and its metabolites (intermediate-density lipoprotein [IDL] and LDL) is decreased in patients with type 2 diabetes, resulting in increased plasma residence time of these lipoproteins and thus further contributing to the dyslipidaemia $[6,7]$.

Traditionally, serum lipids have been studied in terms of the number of VLDL, LDL and HDL particles, and only the largest lipid classes have been quantified in an attempt to further characterise the lipoprotein particle. Although it is well known that the concentration of apolipoprotein (Apo)B-containing lipoproteins (VLDL, IDL and LDL) is a key determinant of atherogenicity, recent data suggest that compositional properties of lipoproteins are also important $[8,9]$. With the advent of powerful analytical tools such as tandem mass spectrometry it is now possible to perform a more detailed characterisation of the different lipoprotein particles. This analysis, which can be performed using a high-throughput approach [10], provides quantitative data about highly abundant lipids as well as the less abundant bioactive lipids. These advances offer an exciting opportunity for the elucidation of mechanisms and for the identification of disease-specific lipid-based biomarkers [11].

In this study, we used a lipidomics approach to characterise lipid classes and lipid species in ApoB-containing lipoproteins isolated from control individuals and normolipidaemic and dyslipidaemic individuals with type 2 diabetes. Our aim was to determine how clinical dyslipidaemia affects the lipoprotein composition in individuals with insulin resistance and type 2 diabetes. We also wanted to investigate if insulin resistance without clinical dyslipidaemia in type 2 diabetes was associated with changes in lipid composition in comparison with a healthy reference group. Finally, we wanted to determine if any of the identified changes could have any biological relevance in the pathophysiology of type 2 diabetes.

\section{Methods}

Lipid annotation For an explanation of lipid annotation, see the electronic supplementary material (ESM).

Study participants The individuals in this study were all 64year-old white women with the same ethnic background (Swedish) who originally participated in the Diabetes and Impaired glucose tolerance in Women and Atherosclerosis (DIWA) study [12]. From this population, we randomly chose 20 women per group according to the following criteria: (1) control individuals (HOMA < 1.35) with normal blood lipids (TAG $<1.7 \mathrm{mmol} / \mathrm{l}$ and HDL-cholesterol $>1.29 \mathrm{mmol} / \mathrm{l}$ ); (2) individuals with normal blood lipids (TAG $<1.7 \mathrm{mmol} / \mathrm{l}$ and HDL-cholesterol $>1.29 \mathrm{mmol} / \mathrm{l}$ ) but with type 2 diabetes as defined by WHO [13], insulin resistance (HOMA >1.35) and glutamic acid decarboxylase antibodies $<4.6 \mathrm{U} / \mathrm{ml}$; and (3) individuals with dyslipidaemia (TAG $>1.7 \mathrm{mmol} / \mathrm{l}$ and HDLcholesterol $<1.29 \mathrm{mmol} / \mathrm{l}$ ), but otherwise the same inclusion criteria as the second group (Table 1). None of the individuals included in the study was using any medication for diabetes or dyslipidaemia. In addition to the three groups above, we also complemented our characterisation with a group of nontreated dyslipidaemic non-diabetic individuals (Table 1). The study protocol was approved by the local ethics committee and each participant provided written informed consent.

Lipoprotein isolation VLDL (including IDL) $(d<1.019)$ and LDL ( $d=1.019-1.063)$ were isolated from $500 \mu$ l plasma by ultracentrifugation [14]. The ApoB concentrations were measured using a Konelab 20 autoanalyser (Thermo Electron, Vantaa, Finland).

LDL sizing using gel electrophoresis LDL particle size was measured using non-denaturing polyacrylamide gel electrophoresis $(2-16 \%$ gradient gels; Alamo, San Antonio, TX, USA) [15]. The gels were stained with Coomassie Brilliant Blue (Merck, Darmstadt, Germany), scanned and analysed using the Quantity One software (Bio-Rad, Richmond, CA, USA). The mean LDL size was measured as described [15].

Lipid extraction Lipids were extracted according to Folch et al [16]. Internal standards were diluted in chloroform and added during the extraction procedure.

Lipid analysis Lipids were analysed using a combination of HPLC and mass spectrometry. For details see the ESM. 
Table 1 Clinical characteristics of the study participants

Values are mean $\pm \mathrm{SD}$

${ }^{*} p<0.05$ vs CRTL; $\uparrow p<0.05$

vs individuals with type 2

diabetes; $₫ p<0.05$ vs individuals

with type 2 diabetes and

dyslipidaemia

T2D, type 2 diabetes; T2D+DL, type 2 diabetes and dyslipidaemia; DL, dyslipidaemia, no diabetes

\begin{tabular}{lcccc}
\hline Characteristic & Controls $(n=20)$ & T2D $(n=20)$ & T2D+DL $(n=20)$ & DL $(n=9)$ \\
\hline BMI $\left(\mathrm{kg} / \mathrm{m}^{2}\right)$ & $24 \pm 3.3$ & $28 \pm 2.7^{*}$ & $32 \pm 4.5^{* \dagger}$ & $29.4 \pm 2.7^{*}$ \\
Waist $(\mathrm{cm})$ & $85 \pm 6.0$ & $96 \pm 8.7^{*}$ & $107 \pm 8.5^{* \dagger}$ & $98 \pm 6.1^{* \dagger}$ \\
TAG (mmol/l) & $1.0 \pm 0.2$ & $1.2 \pm 0.3$ & $2.8 \pm 1.2^{* \dagger}$ & $2.1 \pm 0.3^{* \dagger}$ \\
HDL-cholesterol (mmol/l) & $1.9 \pm 0.2$ & $1.7 \pm 0.3^{*}$ & $1.1 \pm 0.2^{* \dagger}$ & $1.1 \pm 0.1^{* \dagger}$ \\
LDL-cholesterol (mmol/l) & $3.3 \pm 0.7$ & $3.1 \pm 1.1$ & $4.0 \pm 1.0^{\dagger}$ & $4.6 \pm 1.4^{* \dagger}$ \\
HOMA & $0.85 \pm 0.3$ & $3.8 \pm 1.6^{*}$ & $5.4 \pm 3.7^{*}$ & $2.2 \pm 0.9^{\ddagger}$ \\
$\mathrm{HbA}_{1 \mathrm{c}}(\%)$ & $5.5 \pm 0.3$ & $6.2 \pm 0.7^{*}$ & $7.0 \pm 1.6^{*}$ & $5.6 \pm 0.3^{\ddagger}$ \\
$\mathrm{HbA}_{1 \mathrm{c}}(\mathrm{mmol} / \mathrm{mol})$ & $37 \pm 2.9$ & $45 \pm 7.5^{*}$ & $53 \pm 18^{*}$ & $38 \pm 3.6^{\ddagger}$ \\
ApoC-III (g/l) & $0.13 \pm 0.03$ & $0.12 \pm 0.03$ & $0.18 \pm 0.06^{* \dagger}$ & $0.16 \pm 0.04$ \\
C-reactive protein (nmol/l) & $14 \pm 12$ & $23 \pm 36$ & $36 \pm 43$ & $33 \pm 35$ \\
Smoker (n) & 0 & 0 & 0 & 2
\end{tabular}

Cell culture and lipoprotein incubation Fresh human smooth muscle cells (Lonza CC 2571; Lonza, Basel, Switzerland) were seeded onto six-well tissue culture plates after two passages. Cells were cultured in Lonza's special medium cc318s and incubated for $24 \mathrm{~h}$ with VLDL $(45 \mu \mathrm{g} / \mathrm{ml})$ containing either high or low levels of TAG-palmitic acid or in media with both free palmitic acid $(100 \mu \mathrm{mol} / 1)$ [17] and VLDL (45 $\mu \mathrm{g} / \mathrm{ml}$ ) containing low levels of TAG-palmitic acid. All media contained $2 \mathrm{mg} / \mathrm{ml} \mathrm{BSA}$ (fatty acid free and low endotoxin). Cytokine levels in the cell culture medium were analysed with a SECTOR Imager 2400 reader (MesoScale Discovery, Gaithersburg, MD, USA). For further details see the ESM.

Statistical evaluation ANOVA analysis followed by Tukey's post hoc test was used for comparisons between groups. Correlation analysis was performed using the Pearson correlation coefficient. To determine the false discovery rate of large number of tests, $q$ values were calculated from the unadjusted $p$ values using QValue software package for $\mathrm{R}$ [18].

\section{Results}

Basic characteristics of participants The dyslipidaemic type 2 diabetic individuals showed several features of diabetic dyslipidaemia including hypertriacylglycerolaemia and low HDL-cholesterol (Table 1). There was a stepwise significant increase in BMI and waist size between the control, normolipidaemic and dyslipidaemic type 2 diabetic groups but no significant differences in HOMA or $\mathrm{HbA}_{1 \mathrm{c}}$ between the two groups with type 2 diabetes (Table 1). Levels of plasma TAG, HDL-cholesterol and LDL-cholesterol were the same in the normolipidaemic type 2 diabetic and control groups (Table 1).

Dyslipidaemia is required to induce significant alterations in lipoprotein lipid class composition Using a combination of HPLC and mass spectrometry, we quantified eight lipid classes in both VLDL and LDL and showed that the lipid class composition was very similar in lipoproteins from normolipidaemic type 2 diabetic and control individuals (Fig. 1a,b). By contrast, we showed that the VLDL and LDL from dyslipidaemic type 2 diabetic individuals
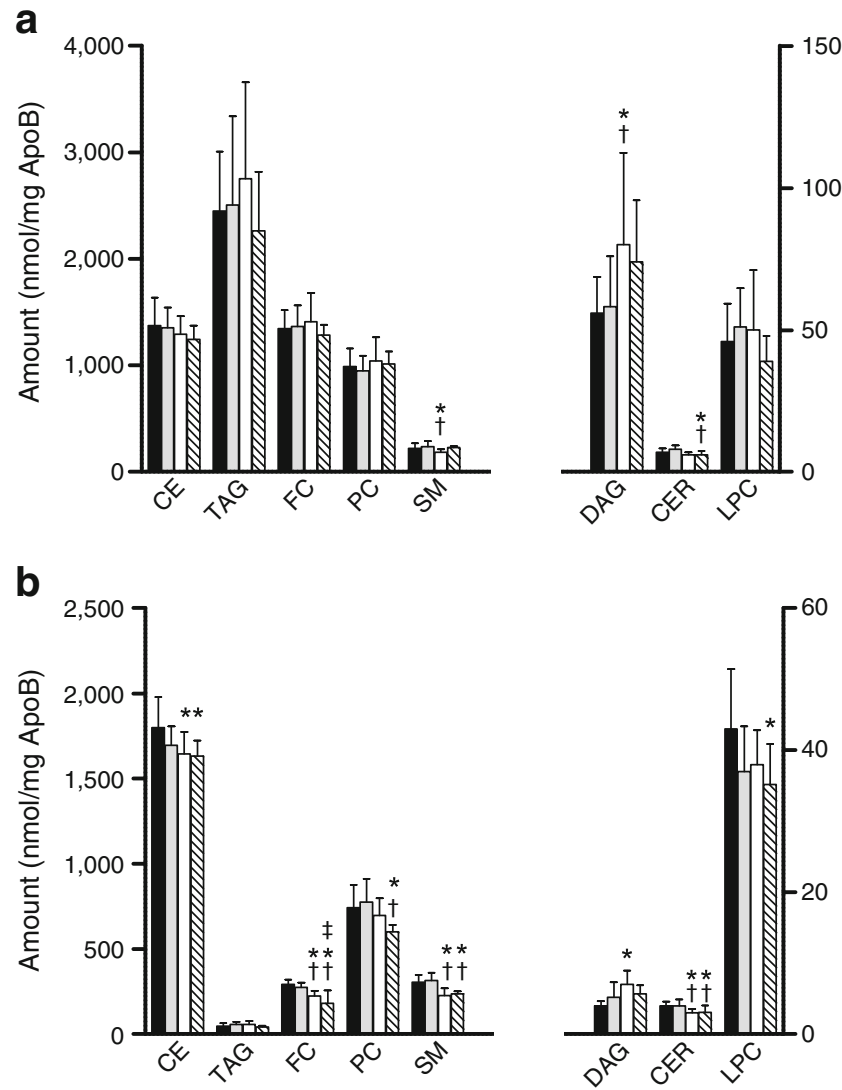

Fig. 1 Lipid class composition of VLDL (a) and LDL (b) isolated from control individuals (black), type 2 diabetic individuals (grey), dyslipidaemic type 2 diabetic individuals (white) and non-diabetic dyslipidaemic individuals (hatched). Values are mean \pm SD. ${ }^{*} p<0.05$ vs control; $\uparrow p<0.05$ vs type 2 diabetic individuals; $₫ p<0.05$ vs dyslipidaemic type 2 diabetic individuals 
displayed significant changes in lipid class composition compared with VLDL and LDL from the other two groups (Fig. 1a,b). In VLDL, diacylglycerol (DAG) was significantly increased and the membrane lipid sphingomyelin (SM) was reduced (Fig. 1a). The changes in LDL were more profound: in parallel with VLDL, we observed increased DAG and decreased SM, but we also measured significantly reduced amounts of the core lipid cholesteryl ester (CE) and the membrane lipids free (non-esterified) cholesterol (FC) and ceramide (CER) (Fig. 1b). The most abundant membrane lipid, phosphatidylcholine (PC), was unchanged, indicating an altered membrane composition.

To test the hypothesis that the compositional differences of LDL are linked to their size, we analysed the correlation between the lipid composition and size of LDL. We showed that LDL isolated from dyslipidaemic type 2 diabetic individuals was smaller than LDL isolated from the normolipidaemic groups (Fig. 2a-h). Furthermore, the size of the LDL correlated negatively with the amount of TAG and DAG and positively with the amount of CE, FC, SM, CER, PC and lysophosphatidylcholine (LPC).

Dyslipidaemia amplifies alterations in PC and CE species composition observed in lipoproteins from type 2 diabetic individuals We comprehensively characterised the lipid species within each lipid class of the isolated lipoproteins using quadrupole time-of-flight (QTOF) mass spectrometry (ESM Table 1). These analyses revealed significantly increased relative amounts of PC 16:0-20:3 (in VLDL and LDL) and PC 18:0-20:3 (in LDL) in lipoproteins from normolipidaemic type 2 diabetic individuals compared with controls (Fig. 3a). These alterations were even more significant in the dyslipidaemic type 2 diabetic group, which also had a relatively increased amount of PC 16:0-16:1 The alterations in the PC fatty acid composition observed in lipoproteins from dyslipidaemic type 2 diabetic participants were reflected in a similar change in the $\mathrm{CE}$ lipid class with significant increases in CE 16:1 (in VLDL and LDL) and CE 20:3 (in LDL) (Fig. 3b). A comparison with basic characteristics showed that the relative amount of PC species containing C16:1 and C20:3 fatty acids correlated significantly with important clinical variables such as BMI and HDL-cholesterol levels (ESM Table 2).

Dyslipidaemia is associated with increased palmitic-acidcontaining species in VLDL and LDL TAG and DAG Mass spectrometric analysis also revealed several significant changes in lipid species in the DAG and TAG fractions of VLDL from dyslipidaemic type 2 diabetic individuals (ESM Table 1; Fig. 4). In DAG, these consisted mainly of increased palmitic acid (C16:0)-containing species such as DAG 16:0-16:0, DAG 16:0-16:1 and DAG 16:0-18:1. Similar patterns were observed in the TAG lipid class with significantly increased palmitic-acid-containing species such as TAG 16:0-16:0-16:0, TAG 16:0-16:0-16:1 and TAG 16:0-16:0-18:1. In contrast, lipid species containing longer and more unsaturated fatty acids, especially linoleic acid (C18:2), were unaltered or decreased (with the exception of an increase in the doubly saturated TAG 16:0-16:018:2 and TAG 18:0-16:0-18:2). All changes detected in VLDL were closely mirrored in the LDL (ESM Table 1). A comparison with basic characteristics revealed several highly significant correlations between molecular TAG and clinical variables (ESM Table 3). A confirmatory GC/flame ionisation detector (FID) analysis of fatty acid methyl esters (FAME) from VLDL TAGs was also performed, which showed similar increases in the relative amount of palmitic acid and reduced amount of linoleic acid (ESM Table 4).

Dyslipidaemia without type 2 diabetes is associated with alterations in VLDL and LDL lipid composition As the results show that dyslipidaemia in type 2 diabetic individuals is associated with an altered VLDL and LDL lipid composition, we also performed a complementary analysis on a group of dyslipidaemic non-diabetic individuals (Table 1). The analysis of the VLDL and LDL lipid class composition showed alterations that were similar to the changes observed for the dyslipidaemic type 2 diabetic individuals when compared with the control group (Fig. 1).

A characterisation of molecular lipid species in the dyslipidaemic non-diabetic individuals showed increased levels of $\mathrm{C} 16: 1$ and $\mathrm{C} 20: 3$ containing species in the CE and PC lipid class compared with the controls. However, the increases were not as pronounced as for the dyslipidaemic type 2 diabetic individuals (Fig. 3). In the TAG and DAG lipid class there was a tendency towards increased levels of palmitic-acid-containing species compared with the control group. However, the results are not as prominent as for the dyslipidaemic type 2 diabetic individuals (Fig. 4).

Dyslipidaemia is linked to increased content of vaccenic acids in a specific molecular TAG Despite the lack of significant alterations of vaccenic acid when analysing total fatty acids using FAME (ESM Table 4), we wanted to investigate whether the increased levels of palmitic acid and palmitoleic acid observed in VLDL TAG from the dyslipidaemic group were reflected by increases in vaccenic acid in specific TAG species. For this we used a combination of collision-induced dissociation (CID) and the novel ozone-induced dissociation (OzID) technology (see ESM for details). The results show that in a specific TAG, individuals with type 2 diabetes have an increased incorporation of the C18:1 fatty acid into the $s n-2$ position (Fig. 5a). Furthermore, dyslipidaemic type 2 diabetic individuals have a higher amount of vaccenic as compared with oleic acid 
Fig. 2 Correlations between lipid class amounts and the size of LDL. All three groups are included in the correlations. a CE, $r=0.58(p<0.01)$; b TAG, $r=0.38(p<0.01) ; \mathbf{c ~ P C}, r=0.46$ $(p<0.01)$; d DAG, $r=0.58$ $(p<0.001)$; e SM, $r=0.49$ $(p<0.001)$; f CER, $r=0.45$ $(p<0.01) ;$ g FC, $r=0.68$ $(p<0.001)$; h LPC, $r=0.52$ $(p<0.001)$. Triangles, controls; circles, normolipidaemic type 2 diabetic individuals; crosses, dyslipidaemic type 2 diabetic individuals
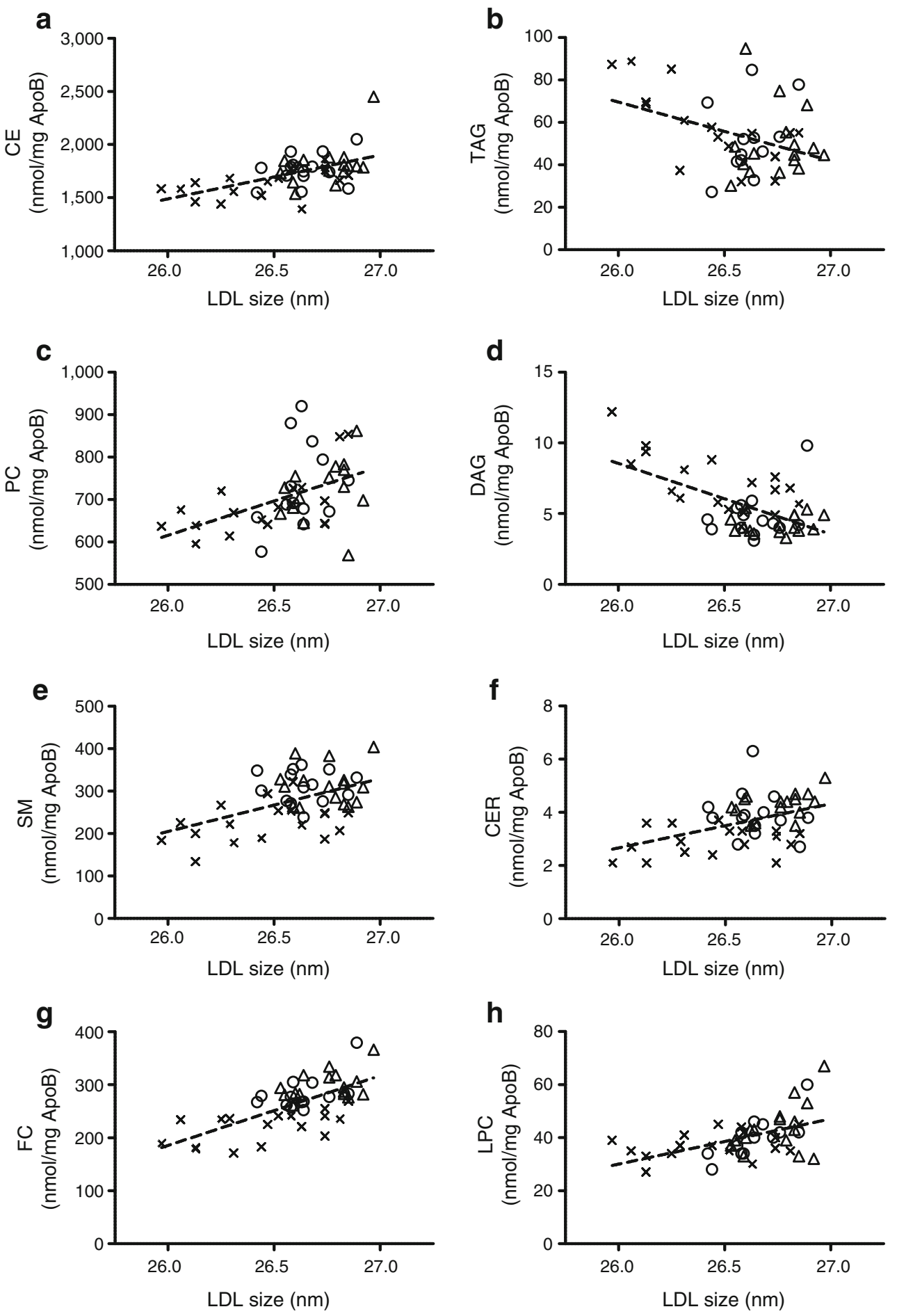

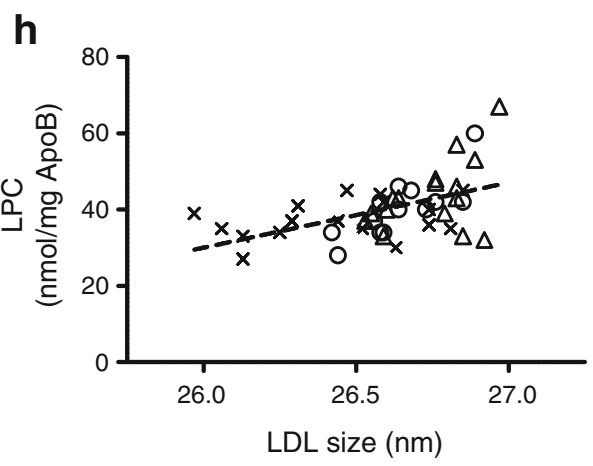

(Fig. 5 b), and this fatty acid is preferably incorporated into the $s n-2$ position of the investigated TAG (Fig. $5 \mathrm{c}$ ).

Increased palmitic acid in VLDL TAG is associated with increased proinflammatory activity To assess the biological significance of the increased palmitic acid in VLDL TAG, we investigated the effect of high and low VLDL TAGpalmitic acid on the inflammatory activity of human smooth muscle cells. We showed that cells incubated with VLDL containing a high proportion of TAG-palmitic acid had a highly significant increase in the secretion of several cytokines involved in endothelial activation (Fig. 6a-i). Cells incubated with a combination of VLDL with low TAGpalmitic acid levels and free palmitic acid (100 $\mu \mathrm{mol} / \mathrm{l})$ also showed increased secretion of inflammatory markers. However, the response was, in most cases, lower than for the 


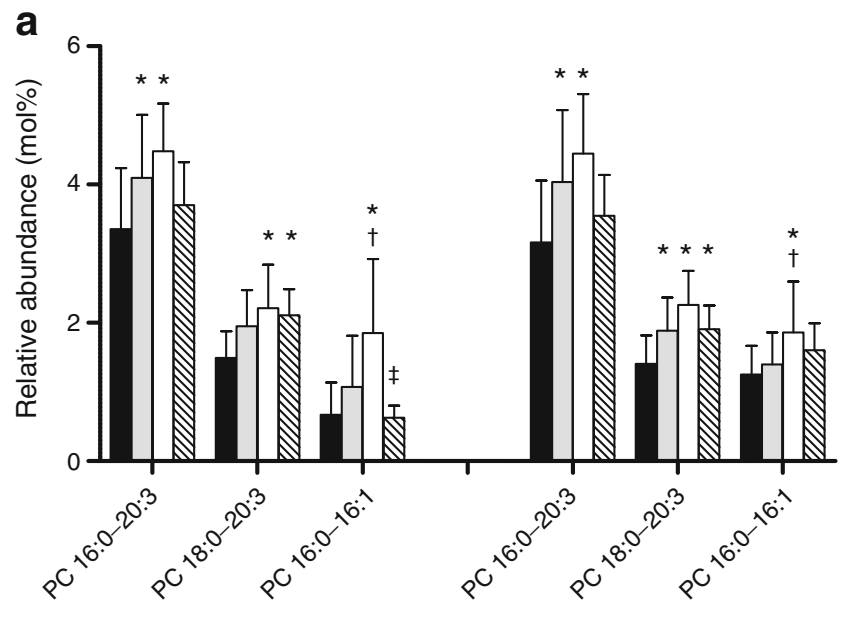

VLDL

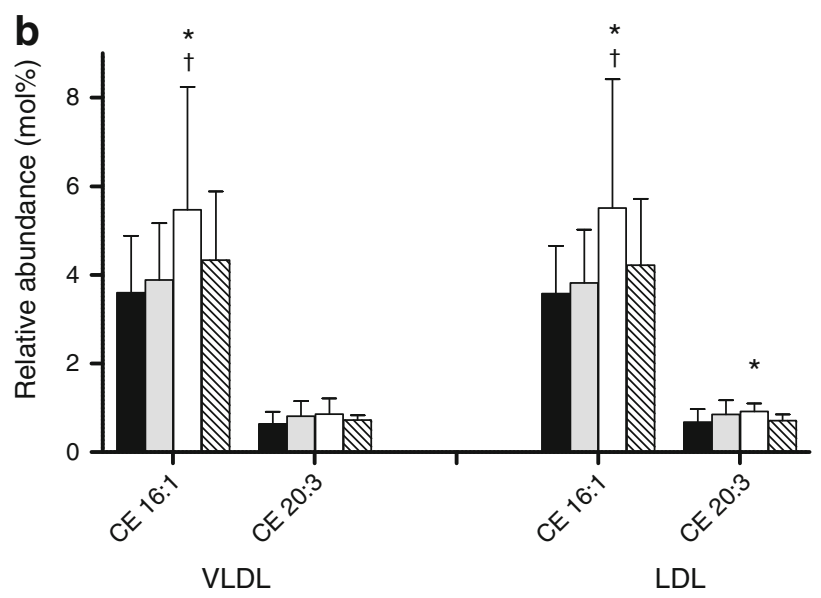

Fig. 3 The relative abundance ( $m o l \%$ of total lipid class) of PC (a) and CE (b) species containing C16:1 and C20:3 fatty acids was compared in control individuals (black), type 2 diabetic individuals (grey), dyslipidaemic type 2 diabetic individuals (white) and non-diabetic dyslipidaemic individuals (hatched). Values are mean \pm SD. ${ }^{*} p<0.05$ vs control; $\uparrow p<0.05$ vs type 2 diabetic individuals; $₫ p<0.05$ vs dyslipidaemic type 2 diabetic individuals

response generated by incubation with VLDL containing high proportions of TAG-palmitic acid.

Assessment of 10 year risk of coronary heart disease from lipid data The 10 year risk for CHD was calculated using the UK Prospective Diabetes Study (UKPDS) risk engine in the type 2 diabetic individuals [19]. Calculation of the UKPDS score is based on blood pressure, $\mathrm{HbA}_{1 \mathrm{c}}$, total cholesterol and HDL-cholesterol as well as age of onset and duration of type 2 diabetes, ethnicity, smoking and sex. Correlations between anthropometric data, blood lipids, selected lipid species and classes and CHD score were calculated (ESM Table 5). By definition, $\mathrm{HbA}_{1 \mathrm{c}}(r=0.82$, $p<0.001)$ and LDL-cholesterol $(r=0.68, p<0.001)$ was positively associated, and HDL-cholesterol negatively associated $(r=-0.62, p<0.001)$ with $\mathrm{CHD}$ risk. In addition, total ApoB $(r=0.90, p<0.001)$, total TAG $(r=0.84, p<0.001)$ and total ApoC-III $(r=0.68, p<0.001)$ showed strong associations with CHD risk. Furthermore, LDL-cholesterol SM $(r=-0.62, p<$ $0.001)$, CER $(r=-0.61, p<0.001), \mathrm{FC}(r=-0.59, p<0.001)$ and DAG $(r=0.56, p<0.001)$ as well as VLDL CER $(r=0.55$, $p<0.01)$ were also associated with CHD risk. Interestingly most of these measures were more strongly associated with CHD risk than HOMA $(r=0.59, p<0.001)$, waist circumference $(r=0.37, p<0.05)$ and BMI $(r=0.26$, NS). Furthermore, using a multivariate approach, combining the levels of several lipids and BMI, waist circumference and HOMA (variables in the UKPDS model [HDL-cholesterol and total cholesterol], or variables highly correlated with these $[\mathrm{ApoB}, \mathrm{ApoA} 1$ and plasma TAG] were excluded), we obtained a high correlation (adjusted $r^{2}=0.72$ ) with the UKPDS score; variables in model were: LDL-cholesterol total CER, waist circumference, VLDL TAG 18:1-18:1-18:1 and VLDL palmitate. Including only lipid variables, we obtained a correlation that was almost as strong (adjusted $r^{2}=0.69$ ); variables in the model were: LDL-cholesterol total CER, LDL DAG 18:0-18:0, VLDL CE 16:1, VLDL total DAG and VLDL DAG 16:1-18:1.

\section{Discussion}

In this study, we used a lipidomics approach to characterise ApoB-containing lipoproteins from control, and from normolipidaemic and dyslipidaemic individuals with type 2 diabetes. We show that dyslipidaemia in individuals with type 2 diabetes is associated with significant changes in specific lipid classes and molecular species in VLDL and LDL. We identify several enriched molecular species in dyslipidaemic VLDL and LDL that correlate with clinical variables and we also show that palmitic-acid-enriched VLDL TAG promotes inflammation.

Small dense LDL is a common characteristic of the dyslipidaemic state [5], and LDL isolated from the participants with dyslipidaemic type 2 diabetes in our study indeed had a smaller particle size compared with LDL from the normolipidaemic participants. A reduced particle size would explain reductions in the membrane lipids CER, SM and FC. However, the most abundant membrane lipid, PC, was unchanged, indicating that LDL would have an altered membrane composition. Because CER, SM and FC have all been shown to positively affect a closer lateral packing in LDL $[20,21]$, the altered lipid composition of LDL isolated from dyslipidaemic type 2 diabetic individuals could be associated with higher membrane fluidity and higher freedom in lateral moving of lipoprotein-associated proteins. Although the metabolic consequences of the reduced lipoprotein SM levels remain to be elucidated, it has been proposed that a decreased SM:PC ratio could render 

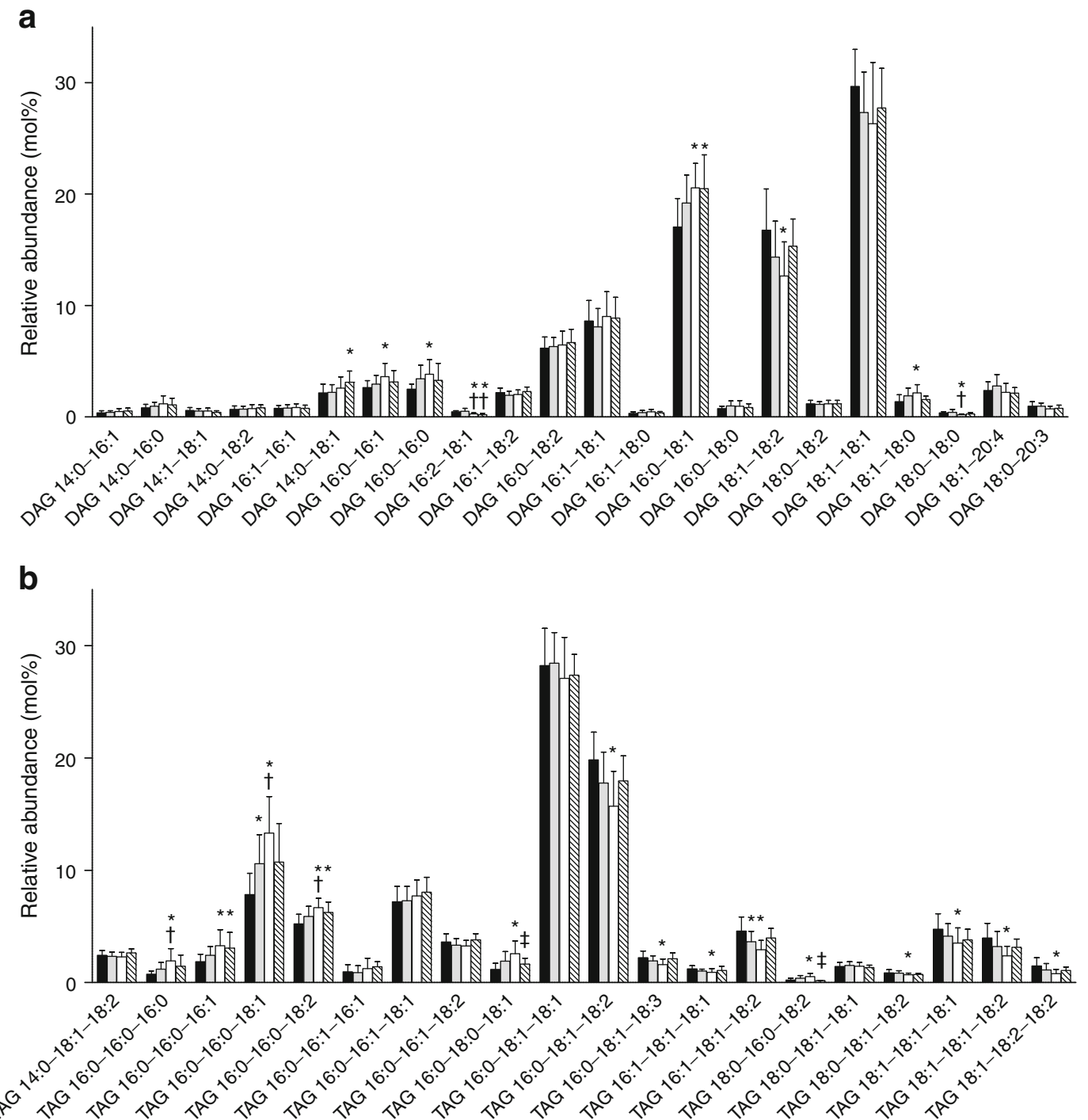

Fig. 4 Lipid species composition of the lipid classes DAG (a) and TAG (b) in VLDL isolated from control individuals (black), type 2 diabetic individuals (grey), dyslipidaemic type 2 diabetic individuals

these particles more susceptible to modifications by phospholipase $\mathrm{A}_{2}$ activity [22, 23].

VLDLs are metabolically heterogeneous, and the liver can secrete both large TAG-rich $\mathrm{VLDL}_{1}$ and smaller cholesterol-rich $\mathrm{VLDL}_{2}$. Variations in plasma TAG concentrations are mainly accounted for by differences in $\mathrm{VLDL}_{1}$. We have earlier shown that increased liver fat in type 2 diabetic individuals is associated with increased $\mathrm{VLDL}_{1}$ production and dyslipidaemia [3]. A possible explanation for the reduced SM/PC, CER/PC and FC/PC ratio observed in dyslipidaemic type 2 diabetic individuals could be a disturbed hepatic lipid metabolism that results in increased secretion of large $\mathrm{VLDL}_{1}$ particles with a different lipid composition from the $\mathrm{VLDL}_{2}$ particle.

Another explanation for the altered membrane composition is that lipoproteins from type 2 diabetic individuals with (white) and non-diabetic dyslipidaemic individuals (hatched). Values are mean \pm SD. ${ }^{*} p<0.05$ vs control; $\dagger p<0.05$ vs dyslipidaemic type 2 diabetic individuals; $₫ p<0.05$ vs type 2 diabetic individuals

dyslipidaemia are potentially more susceptible to modifications occurring in the circulation. Studies have shown that sphingomyelinase (SMase) activity is elevated in the serum of patients with type 2 diabetes [24], and also that LDL isolated from type 2 diabetic individuals is enriched with the SMase activator ApoC-III [25]. Depletion of cell-surface SM with SMase results in a simultaneous loss of FC [26], and thus increased SMase activity will result in parallel decreases in SM and FC.

Although TAG was slightly increased in VLDL and LDL isolated from dyslipidaemic type 2 diabetic individuals, the differences were not significant. We expected to see greater changes in TAG, but it is likely that large TAG-rich VLDL characteristic of dyslipidaemic individuals is rapidly metabolised to TAG-poor VLDL. Furthermore, our VLDL fraction did not differentiate between VLDL and IDL and therefore 


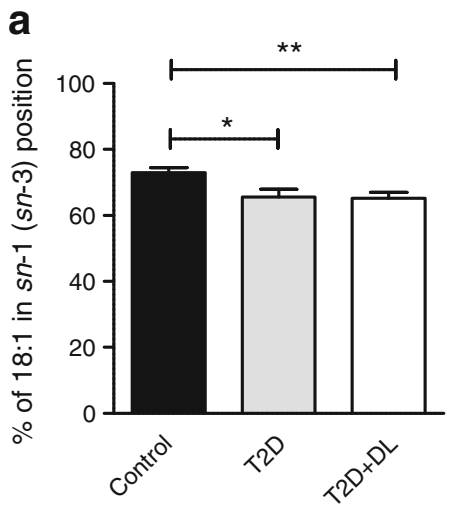

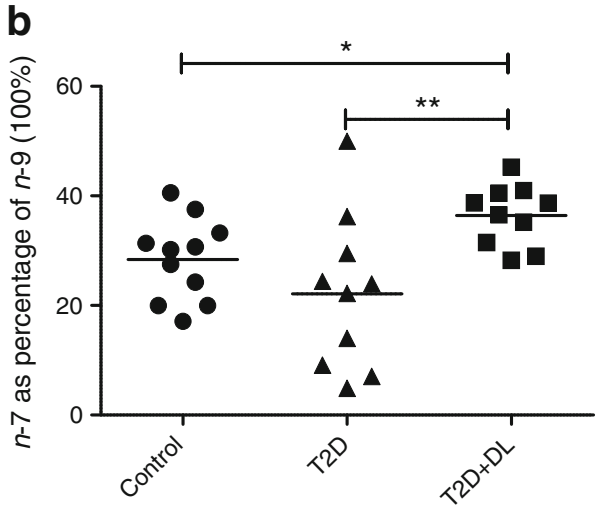

TAG 50:1

(OzID)

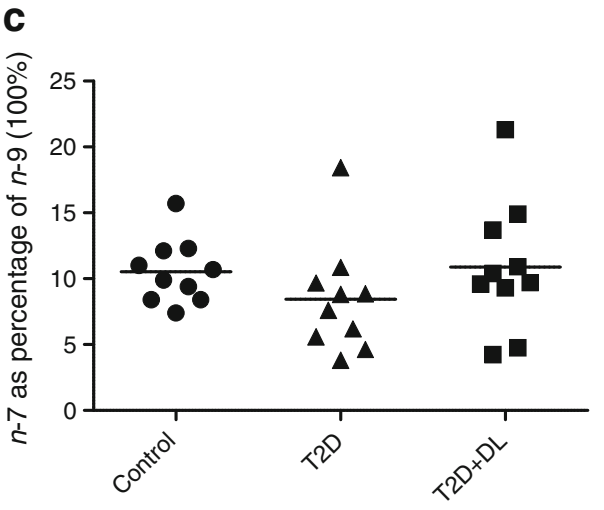

TAG 16:0-16:0-18:1 $(\mathrm{CID} / \mathrm{OzID})^{2}$
Fig. 5 a Using CID, the ratio of the molecular species TAG 16:0 16:0-18:1 (C18:1 in $s n-1$ [3] position) and the isomer TAG 16:0-18:116:0 (C18:1 in $s n-2$ position) were determined in the VLDL TAGs isolated from control individuals (black), normolipidaemic type 2 diabetic individuals (grey), and individuals with type 2 diabetes and dyslipidaemia (white) ( $n=10$ in each group). Values are mean \pm SD. b,c Using combinations of CID and OzID the ratio of vaccenic (C18:1 $n-7)$ to

some of the changes in TAG may have been masked. By contrast, we observed clear increases in DAG in VLDL and LDL isolated from dyslipidaemic type 2 diabetic individuals. A role for DAGs has been implicated in the aetiology of insulin resistance in the liver through a mechanism involving activation of protein kinase $C-\varepsilon[27,28]$. However, it remains oleic (C18:1 n-9) acid was determined in the molecular species TAG 50:1 (b) and TAG 16:0-16:0-18:1 (c). This was done in VLDL TAG isolated from control individuals, normolipidaemic individuals with type 2 diabetes, and individuals with dyslipidaemia and type 2 diabetes $(n=10$ in each group). ${ }^{*} p<0.05 ;{ }^{*} p<0.01$. DL, individuals with dyslipidaemia; T2D, individuals with type 2 diabetes

to be determined if hepatic uptake of DAG-enriched lipoproteins could contribute to this pathway.

We also showed that the PC lipid class in VLDL and LDL isolated from dyslipidaemic type 2 diabetic individuals had increased levels of $\mathrm{C} 16: 1$ and $\mathrm{C} 20: 3$ that were reflected in the CE. These parallel increases in PC and CE may be
Fig. 6 Cells were incubated with VLDL containing either high (High) or low (Low) levels of palmitic acid in the TAG, or with a combination of VLDL containing low levels of palmitic acid and free palmitic acid $($ Low $+\mathrm{P})$. The cytokine concentrations $(\mathrm{pg} / \mathrm{ml})$ were measured in the cell media and are expressed as mean $\pm \mathrm{SD}$ $(n=5) . * p<0.05 ; * * p<0.01$; $* * * p<0.005$
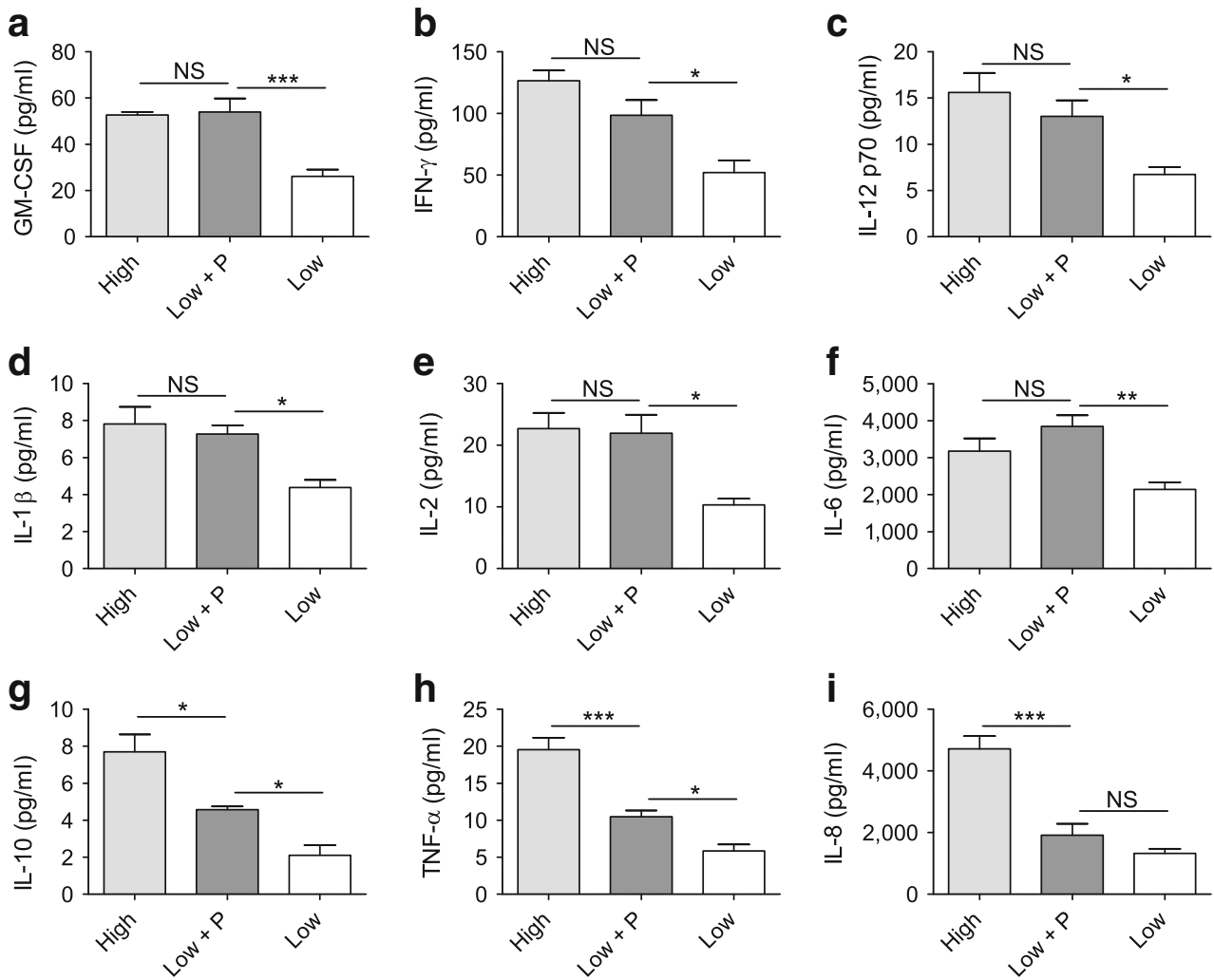
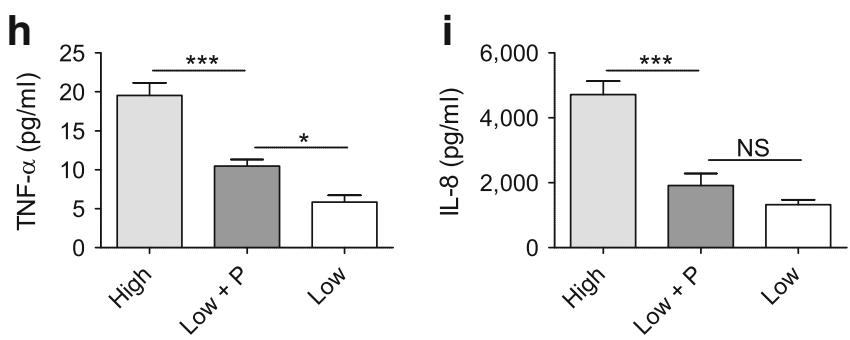
explained by the fact that $\mathrm{CE}$ can be synthesised by the lecithin-cholesterol acyltransferase-catalysed transesterification of FC with the fatty acid attached to the $s n-2$ position of PC [29]. Increased levels of C16:1 and C20:3 esterified to $\mathrm{PC}$ and $\mathrm{CE}$ have previously been observed in individuals with type 2 diabetes [30,31], and here we show that the relative amount of $\mathrm{PC}$ and $\mathrm{CE}$ species containing C16:1 and C20:3 correlated significantly with clinical variables.

Analysis of the lipid species composition of the DAG and TAG lipid classes revealed that VLDL and LDL from dyslipidaemic type 2 diabetic individuals were enriched in palmitic acid (C16:0) whereas linoleic acid (C18:2) was mainly unaffected or reduced. A recent study showed that serum TAGs containing palmitic acid correlates positively with insulin resistance whereas serum TAGs containing linoleic acid correlate negatively with insulin resistance [32]. Furthermore, they also showed that TAG 16:0-16:018:1 and TAG 16:0-18:1-18:0 are better markers of insulin resistance than total serum TAG concentration [32]. We also showed that palmitic acid in TAG from VLDL and LDL correlated positively with insulin resistance whereas linoleic acid correlated negatively. However, both of these correlations were weaker than the correlation between total plasma TAG and insulin resistance in our study.

Using a combination of CID and the novel OzID techniques, we showed an elevation of vaccenic acid in dyslipidaemic type 2 diabetic individuals, specifically in TAG 16:0-16:0-18:1. Furthermore, our results suggested that the $s n-2$ position of the glycerol backbone is the preferred position for its incorporation, which is in accordance with previously published data [33]. As hepatic fat accumulation is associated with dyslipidaemia in type 2 diabetes [34], we hypothesise that the increased content of vaccenic acid is an indication of altered hepatic lipid metabolism and a marker of hepatic steatosis. However, the reason for the selective incorporation into specific TAG or its potential role as a biomarker demands further investigation.

In addition to TAG 16:0-16:0-18:1, which was the TAG species that was most significantly changed between the dyslipidaemic type 2 diabetic individuals and controls, we also did a similar characterisation of the most common TAG species, TAG 18:1-18:1-16:0. Here we saw no alteration in the $n-7: n-9$ ratio. This result might be the reason for not detecting a difference in $n-7: n-9$ ratio when analysing FAME using GC/FID. In that analysis, selective fatty acid incorporation into certain molecular TAG species will be masked by the signal from highly abundant, non-changing, C18:1 $n$-7 and C18:1 $n-9$ containing TAG species (e.g. TAG 18:1-18:1-16:0).

It has previously been shown that VLDL isolated from individuals with dyslipidaemia is proinflammatory and responsible for the upregulation of several pathways involved in endothelial activation [35]. Although the mechanisms behind this are poorly understood, the composition of the particle might play an important role. For example, TAGrich lipoproteins (TRLs) isolated after a meal are more proinflammatory if enriched in saturated fatty acids rather than mono- and polyunsaturated fatty acids [36]. Furthermore, palmitic acid (in its free form) has been shown to be bioactive and proinflammatory $[37,38]$. Because the TAG in the VLDL particles isolated from dyslipidaemic individuals in our study was enriched in palmitic acid, we tested the hypothesis that this would increase the proinflammatory properties of these particles. Incubation of smooth muscle cells with VLDL rich in TAG-palmitic acid resulted in a highly significant increase in the secretion of several important inflammatory mediators. However, although this supports our theory, we cannot exclude the possibility that other intrinsic factors of the VLDL particle could be responsible for the results.

In an attempt to evaluate the clinical relevance of our results, we correlated several of the lipid variables to CHD risk as calculated by the UKPDS engine. Interestingly, the analysis shows that many of these correlations were more strongly associated with CHD risk than traditional risk markers such as HOMA, waist and BMI. Furthermore, even stronger correlations could be obtained if several lipids were used in a multivariate approach. However, as this is not a definite estimate, further studies involving a larger cohort are needed to evaluate the importance of these variables compared with the traditional risk factors.

We also performed a complementary analysis of lipoproteins isolated from a group of dyslipidaemic individuals with normal glucose tolerance, with the aim of further clarifying the role of dyslipidaemia for the observed changes in lipoprotein lipid composition. However, such a comparison was hampered by the fact that less than $5 \%(n=9)$ of the individuals with normal glucose tolerance and no lipidlowering treatment fulfilled the dyslipidaemia criteria. Both hypertriacylglycerolaemia and low HDL-cholesterol are well-known predictors of diabetes [39]. Furthermore, all individuals had abdominal obesity with waist girth above $88 \mathrm{~cm}$, seven out of nine individuals had insulin resistance, and $56 \%(n=5)$ had a first-degree relative with diabetes, compared with $18 \%(n=33, p=0.016)$ in those without dyslipidaemia. As all the mentioned characteristics are strong predictors of type 2 diabetes, it is obvious that these individuals with dyslipidaemia represent a group with prediabetes, albeit still without hyperglycaemia. This conclusion is supported by the striking similarity between the two dyslipidaemic groups with and without diabetes regarding obesity, degree of dyslipidaemia, and levels of C-reactive protein and ApoC-III (Table 1). In line with these observations we found that, overall, the lipid aberration in the dyslipidaemic non-diabetic group was similar to that in the dyslipidaemic type 2 diabetic group, although not as pronounced. Taken together these data support previous 
suggestions that dyslipidaemia may precede the development of type 2 diabetes.

A possible limitation of our study is that only women of a certain age are included. Therefore it is not obvious that these results can be directly translated to the male population. However, the cohort of women that was investigated in the present study is of considerable interest given that diabetes prevalence starts to increase steeply in this age category in women [40]. In addition, the diagnosis of diabetes is associated with a very high relative risk of future cardiovascular death in women that is much higher than among men [41].

Acknowledgements The authors would like to thank M. Heyden for excellent technical assistance and R. Perkins for editorial expertise.

Funding This work was supported by the Swedish Research Council, the Swedish Heart-Lung Foundation, the Novo Nordisk Foundation, Torsten and Ragnar Söderberg Foundation, Swedish Foundation for Strategic Research and the EU-funded projects EtherPaths and Lipidomic Net.

Contribution statement MS performed the lipid analyses, interpreted the data and wrote the manuscript. HTP, TWM and SJB performed lipid analysis, contributed to the data interpretation and the draft of the manuscript. MA performed the statistical analysis and interpreted the data. KE contributed to the study conception and design, and also to the draft of the manuscript. JB and BF contributed to the study conception and design, data analysis and interpretation, and draft of the manuscript. All authors revised the manuscript critically for intellectual content and approved of the final version.

Duality of interest The authors confirm that there is no duality of interest associated with this manuscript.

\section{References}

1. Haffner SM, Lehto S, Ronnemaa T, Pyorala K, Laakso M (1998) Mortality from coronary heart disease in subjects with type 2 diabetes and in nondiabetic subjects with and without prior myocardial infarction. N Engl J Med 339:229-234

2. Saydah SH, Fradkin J, Cowie CC (2004) Poor control of risk factors for vascular disease among adults with previously diagnosed diabetes. JAMA 291:335-342

3. Adiels M, Taskinen MR, Packard C et al (2006) Overproduction of large VLDL particles is driven by increased liver fat content in man. Diabetologia 49:755-765

4. Adiels M, Westerbacka J, Soro-Paavonen A et al (2007) Acute suppression of VLDL1 secretion rate by insulin is associated with hepatic fat content and insulin resistance. Diabetologia 50:23562365

5. Taskinen MR (2003) Diabetic dyslipidaemia: from basic research to clinical practice. Diabetologia 46:733-749

6. Chan DC, Watts GF, Redgrave TG, Mori TA, Barrett PH (2002) Apolipoprotein B-100 kinetics in visceral obesity: associations with plasma apolipoprotein C-III concentration. Metabolism 51:1041-1046

7. Riches FM, Watts GF, Naoumova RP, Kelly JM, Croft KD, Thompson GR (1998) Hepatic secretion of very-low-density lipoprotein apolipoprotein B-100 studied with a stable isotope technique in men with visceral obesity. Int $\mathrm{J}$ Obes Relat Metab Disord 22:414-423

8. Kontush A, Chapman MJ (2010) Lipidomics as a tool for the study of lipoprotein metabolism. Curr Atheroscler Rep 12:194-201

9. Sysi-Aho M, Vehtari A, Velagapudi VR et al (2007) Exploring the lipoprotein composition using Bayesian regression on serum lipidomic profiles. Bioinformatics 23:i519-i528

10. Stahlman M, Ejsing CS, Tarasov K, Perman J, Boren J, Ekroos K (2009) High-throughput shotgun lipidomics by quadrupole timeof-flight mass spectrometry. J Chromatogr B Analyt Technol Biomed Life Sci 877:2664-2672

11. Ekroos K, Janis M, Tarasov K, Hurme R, Laaksonen R (2010) Lipidomics: a tool for studies of atherosclerosis. Curr Atheroscler Rep 12:273-281

12. Brohall G, Behre CJ, Hulthe J, Wikstrand J, Fagerberg B (2006) Prevalence of diabetes and impaired glucose tolerance in 64-yearold Swedish women: experiences of using repeated oral glucose tolerance tests. Diabetes Care 29:363-367

13. World Health Organization (1999) Definition, diagnosis and classification of diabetes mellitus and its complications. Report of WHO Consultation. Part I: diagnosis and classification of diabetes mellitus. World Health Organization, Geneva

14. Stahlman M, Davidsson P, Kanmert I et al (2008) Proteomics and lipids of lipoproteins isolated at low salt concentrations in $\mathrm{D}_{2} \mathrm{O}$ / sucrose or in KBr. J Lipid Res 49:481-490

15. Hulthe J, Wiklund O, Olsson G et al (1999) Computerized measurement of LDL particle size in human serum. Reproducibility studies and evaluation of LDL particle size in relation to metabolic variables and the occurrence of atherosclerosis. Scand J Clin Lab Investig 59:649-661

16. Folch J, Lees M, Sloane Stanley GH (1957) A simple method for the isolation and purification of total lipides from animal tissues. $\mathrm{J}$ Biol Chem 226:497-509

17. Haversen L, Danielsson KN, Fogelstrand L, Wiklund O (2009) Induction of proinflammatory cytokines by long-chain saturated fatty acids in human macrophages. Atherosclerosis 202:382393

18. Storey JD, Tibshirani R (2003) Statistical significance for genomewide studies. Proc Natl Acad Sci USA 100:9440-9445

19. Stevens RJ, Kothari V, Adler AI, Stratton IM (2001) The UKPDS risk engine: a model for the risk of coronary heart disease in type II diabetes (UKPDS 56). Clin Sci (Lond) 101:671-679

20. Ibdah JA, Lund-Katz S, Phillips MC (1989) Molecular packing of high-density and low-density lipoprotein surface lipids and apolipoprotein A-I binding. Biochemistry 28:1126-1133

21. Sola R, Baudet MF, Motta C, Maille M, Boisnier C, Jacotot B (1990) Effects of dietary fats on the fluidity of human high-density lipoprotein: influence of the overall composition and phospholipid fatty acids. Biochim Biophys Acta 1043:43-51

22. Gesquiere L, Cho W, Subbaiah PV (2002) Role of group IIa and group $\mathrm{V}$ secretory phospholipases $\mathrm{A}(2)$ in the metabolism of lipoproteins. Substrate specificities of the enzymes and the regulation of their activities by sphingomyelin. Biochemistry 41:4911-4920

23. Pettersson C, Fogelstrand L, Rosengren B et al (2008) Increased lipolysis by secretory phospholipase $\mathrm{A}(2)$ group $\mathrm{V}$ of lipoproteins in diabetic dyslipidaemia. J Intern Med 264:155-165

24. Gorska M, Baranczuk E, Dobrzyn A (2003) Secretory $\mathrm{Zn}^{2+}$-dependent sphingomyelinase activity in the serum of patients with type 2 diabetes is elevated. Horm Metab Res 35:506-507

25. Hiukka A, Stahlman M, Pettersson C et al (2009) ApoCIIIenriched LDL in type 2 diabetes displays altered lipid composition, increased susceptibility for sphingomyelinase, and increased binding to biglycan. Diabetes 58:2018-2026

26. Ohvo H, Olsio C, Slotte JP (1997) Effects of sphingomyelin and phosphatidylcholine degradation on cyclodextrin-mediated 
cholesterol efflux in cultured fibroblasts. Biochim Biophys Acta 1349:131-141

27. Shmueli E, Alberti KG, Record CO (1993) Diacylglycerol/protein kinase $\mathrm{C}$ signalling: a mechanism for insulin resistance? J Intern Med 234:397-400

28. Nagle CA, An J, Shiota M et al (2007) Hepatic overexpression of glycerol-sn-3-phosphate acyltransferase 1 in rats causes insulin resistance. J Biol Chem 282:14807-14815

29. Glomset JA (1962) The mechanism of the plasma cholesterol esterification reaction: plasma fatty acid transferase. Biochim Biophys Acta $65: 128-135$

30. Vessby B, Aro A, Skarfors E, Berglund L, Salminen I, Lithell H (1994) The risk to develop NIDDM is related to the fatty acid composition of the serum cholesterol esters. Diabetes 43:1353-1357

31. Pelikanova T, Kazdova L, Chvojkova S, Base J (2001) Serum phospholipid fatty acid composition and insulin action in type 2 diabetic patients. Metabolism 50:1472-1478

32. Kotronen A, Velagapudi VR, Yetukuri L et al (2009) Serum saturated fatty acids containing triacylglycerols are better markers of insulin resistance than total serum triacylglycerol concentrations. Diabetologia 52:684-690

33. Reichwald-Hacker I, Kiewitt I, Ilsemann K, Mukherjee KD (1979) Vaccenic acid in tissue lipids and its positional distribution in glycerolipids of rats fed a polyunsaturated fat diet. J Nutr 109:565-572

34. Adiels M, Olofsson SO, Taskinen MR, Boren J (2008) Overproduction of very low-density lipoproteins is the hallmark of the dyslipidemia in the metabolic syndrome. Arterioscler Thromb Vasc Biol 28:1225-1236

35. Norata GD, Grigore L, Raselli S et al (2006) Triglyceride-rich lipoproteins from hypertriglyceridemic subjects induce a proinflammatory response in the endothelium: molecular mechanisms and gene expression studies. J Mol Cell Cardiol 40:484-494

36. Williams CM, Maitin V, Jackson KG (2004) Triacylglycerol-rich lipoprotein-gene interactions in endothelial cells. Biochem Soc Trans 32:994-998

37. El-Assaad W, Buteau J, Peyot ML et al (2003) Saturated fatty acids synergize with elevated glucose to cause pancreatic beta-cell death. Endocrinology 144:4154-4163

38. Maedler K, Oberholzer J, Bucher P, Spinas GA, Donath MY (2003) Monounsaturated fatty acids prevent the deleterious effects of palmitate and high glucose on human pancreatic beta-cell turnover and function. Diabetes 52:726-733

39. D'Agostino RB Jr, Hamman RF, Karter AJ, Mykkanen L, Wagenknecht LE, Haffner SM (2004) Cardiovascular disease risk factors predict the development of type 2 diabetes: the insulin resistance atherosclerosis study. Diabetes Care 27:2234-2240

40. The DECODE Study Group (2003) Age- and sex-specific prevalences of diabetes and impaired glucose regulation in 13 European cohorts. Diabetes Care 26:61-69

41. Niskanen L, Turpeinen A, Penttila I, Uusitupa MI (1998) Hyperglycemia and compositional lipoprotein abnormalities as predictors of cardiovascular mortality in type 2 diabetes: a 15 -year follow-up from the time of diagnosis. Diabetes Care 21:1861-1869 